\title{
Shear-affected depletion interaction
}

\author{
C. July ${ }^{1, a}$, D. Kleshchanok ${ }^{2}$, and P.R. Lang ${ }^{1, b}$ \\ 1 Forschungszentrum Jülich, ICS-3 - Soft Matter, Jülich, Germany \\ 2 Van 't Hoff Laboratory for Physical and Colloid Chemistry - Debye Research Institute, Utrecht University, The Netherlands
}

Received 17 January 2012 and Received in final form 8 May 2012

Published online: 19 July 2012

(C) The Author(s) 2012. This article is published with open access at Springerlink.com

\begin{abstract}
We investigate the influence of flow fields on the strength of the depletion interaction caused by disc-shaped depletants. At low mass concentration of discs, it is possible to continuously decrease the depth of the depletion potential by increasing the applied shear rate until the depletion force is not perceivable experimentally. Above a threshold in the platelet mass concentration, the depletion potential can no longer be affected by flow in the accessible range of shear rates. While the observed decrease of depletion strength at low depletant concentration may be ascribed to flow alignment of the discs, it is not clear why the influence of flow is vanishing at high concentrations. In order to observe these effects, a modification of the established total internal reflexion microscopy (TIRM) technique is be implemented. We show the suitability of these modifications to measure particle-wall interaction potentials under non-equilibrium conditions for systems where particles are exposed to a shear.
\end{abstract}

\section{Introduction}

In most technical applications of colloidal or polymer solutions, and in many biological systems, various components of different size and shape are dispersed in a common solvent. This gives rise to a very complex system of mutual interactions due to excluded-volume effects, which are usually referred to as depletion interactions [1] or as crowding effects in the field of cell biology. In the simplest case these interactions arise if two types of colloidal particles of different size or shape are mixed in a suspension [2]. They originate from an osmotic pressure imbalance due to expulsion of smaller particles from the gaps, formed upon approach between the larger particle species or large particles and the walls of the container. Since the gaps have to be of the same dimension as the depleted particles, the so-called depletants, it is obvious that depletion attraction has a finite range, which depends on the dimension of the depletant. The depletion strength, aside from size and shape of the depletants, is mainly governed by the particle number density. Thus, by choice of a suitably shaped depletant and variation of depletant size and concentration, a depletion potential may be tailored and tuned to a desired outcome. This offers an effective route to manipulate macroscopic properties of colloidal suspensions such as their rheology, structure formation and phase behavior [36 , since these properties are dictated by the effective particle interaction potential. In this paper we investigate

\footnotetext{
a e-mail: e.july@fz-juelich.de

b e-mail: p.lang@fz-juelich.de
}

whether it is possible to change depletion potentials by application of external stimuli, which for technical applications might be much easier than changing the depletant concentration or size. The effect of temperature on the depletion interaction has already been studied by Gregory et al. with poly-N-isopropylacrylamide (PNIPAM) hydrogel particles as a thermosensitive depletant [7]. Their work shows the possibility to induce crystallization by changing the depletion interaction mediated by a temperature change. Similarly, electrical and magnetic fields might be employed, if the depletant particles were susceptible. In this study we choose flow fields to exert an external force on disc-like depletants, with the aim to shear-align them and thereby manipulating the range and the strength of the depletion potential. This choice is motivated by the fact that a flow field can be realized relatively easily. Further, many systems in which depletants play a role, such as drilling fluids (where clays are used as rheological modifiers) or red blood cells inside the blood stream of an organism [8], are exposed to shear gradients. Therefore, it is highly desirable to improve our understanding of the interrelation between a shear gradient and the effective particle interaction.

The problem of manipulating depletion forces by flow has been treated theoretically by Dzubiella et al. [9], who showed that it is possible in principle to tune depletion forces by variation of the flow velocity. However, experimental investigations on flowing systems are not available, although the depletion interaction in quiescent suspensions has been studied experimentally in great detail for various types of depletants. For this purpose, optical 
tweezers techniques [10] or in most of the cases total internal reflection microscopy (TIRM) [11-19] was applied. Only these two methods provide the sensitivity which is necessary to measure interaction forces as weak as those between colloidal particles. Consequently, we employ a modified TIRM method, which we describe in detail in the experimental section. In brief (for a detailed description the interested reader is referred to the pioneering work of D. Prieve [20]) the standard TIRM works as follows: a probe sphere, which has to reside in a shallow potential minimum (usually provided by the superposition of gravity and electrostatic repulsion) at an average distance of about $100 \mathrm{~nm}$ away from a flat wall, is illuminated with the evanescent field of a totally reflected laser beam. The evanescent field is characterized by its penetration depth $\beta^{-1}$, at which the field intensity has decayed to $1 / e$ of its value at the interface. The intensity of the light, which the probe sphere scatters, fluctuates in time because the illuminating field strength decays exponentially with distance from the wall, and the particle moves up and down along the surface normal due to Brownian motion. Since the scattered intensity is, to a very good approximation, proportional to the square of the evanescent field strength, the probability to observe a certain scattered intensity is equal to the probability of finding the sphere at the corresponding height. Therefore the histogram of observed scattering intensities can be converted into the probability density distribution of separation distances between the sphere and the wall which can be used to calculate the sphere-wall interaction potential via Boltzmann's law. To obtain reliable results, the bins of the intensity histograms have to be small enough to justify the transition from a histogram to a smooth distribution, and the number of events in each bin has still to be large enough to provide satisfying statistics. This limit requires that the probe sphere can be observed on the order of ten to fifteen minutes in an experiment, which will not be possible if the probe sphere is subject to a flow field.

Our modified method relies on video microscopy and tracking of a multitude of probe spheres moving through the microscope's field of view in contrast to the standard TIRM experiment, in which the intensity of the light scattered by a single probe-sphere, usually kept in place by optical tweezers, is detected with a photomultiplier tube (PMT) [20].

The shear gradients in our experiments are introduced by using a thin flow cell, while the flow is driven by gravity and regulated by an electronic valve. It is not clear that for this experimental situation Boltzmann's law may be applied to calculate the interaction potential, because the system is not in thermal equilibrium. We will, however, provide theoretical arguments in favor of this approach. Further, we show experimentally that when no depletants are present a shear field has no influence on the interaction potential between the probe sphere and the wall.

We perform experiments on silica-coated gibbsite platelets as depletants [21], because the depleted colloid has to exhibit some asymmetry in order to be aligned by a shear field. The platelets used are comparatively monodisperse particles and their depletion behavior is theoretically

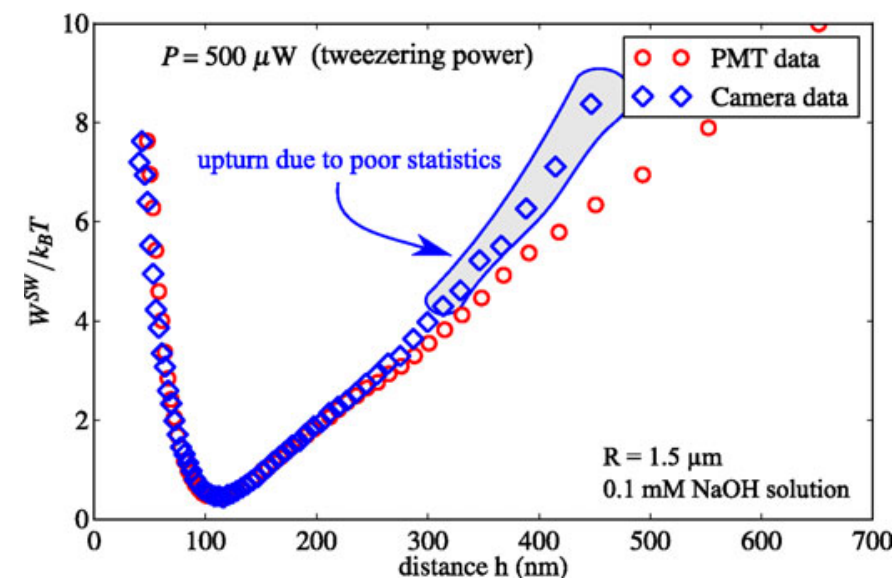

Fig. 1. Comparison between interaction potentials measured using two different detecting units, as indicated. Data were collected from a $R=1.5 \mu \mathrm{m}$ polystyrene bead in $1 \mathrm{mM} \mathrm{NaOH}$ solution.

predictable [22]. They have a hexagonal shape and a thickness significantly smaller than the diameter of the circle enclosing them. Their inorganic nature facilitates sample handling of the platelets. In contrast to biological samples such as fd virus $[23,15]$ (a common model system for rod shaped depletants), these platelets require almost no special $p \mathrm{H}$ conditions and are stable in a broad spectrum of solvents [24,25].

Our experimental data show that the depletion interaction between spherical probes and a flat wall induced by platelets weakens with increasing shear rate. We interpret this observation as being the result of the platelets being aligned in the shear gradient. Shear alignment reduces the effective dimension of the depleted colloids, which in turn decreases the range and the magnitude of the depletion effect.

\section{Experimental}

\subsection{Microscope setup and multi particle measurements}

The TIR microscope, which is the basis for the modified TIRM setup, was self-built from standard microscopy components (Olympus), as described in detail in an earlier contribution [22]. For the experiments presented here, a $15 \mathrm{~mW}$ HeNe laser $\left(\lambda_{\mathrm{ill}}=632 \mathrm{~nm}\right.$, noise $\left.\mathrm{RMS}<0.20 \%\right)$ is used as an illumination source. Although standard TIRM is the method of choice to measure sphere-wall interaction potentials, it suffers from two severe limitations. The intensity trace from only one particle can be measured at a time and measurements are only possible if the probe particle does not move laterally. When employing a highly sensitive electron multiplying charge-coupled device (EMCCD) camera these restrictions are removed. However, comparative potential measurements show that the range over which potential profiles obtained with the camera agree with those measured using the photomultiplier tube is limited. In fig. 1 we compare the potential between 

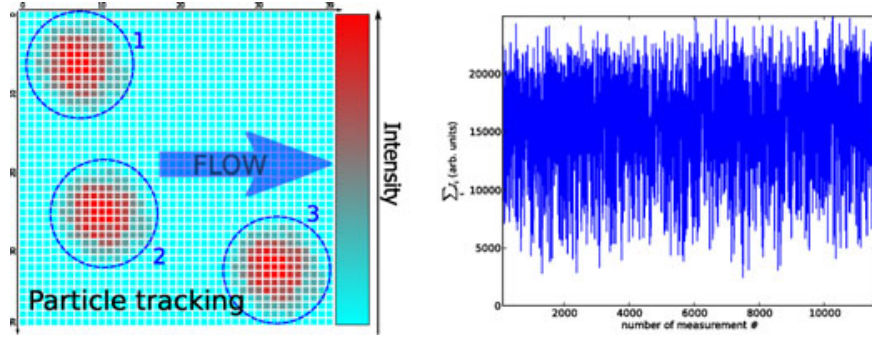

Fig. 2. Illustration of data acquisition with the EMCCDcamera from a multitude of probe spheres. When measuring with the camera, particle tracking is performed to acquire the individual intensity trace of each particle. To process the data and convert them into an interaction potential, the single particle intensity traces are summed up to give an effective single particle trace.

a single polystyrene sphere in a quiescent fluid and the glass wall, which was measured with the camera to the corresponding potential obtained simultaneously with the standard technique. The exponentially decaying branch at small distances, which is due to electrostatic repulsion, and the the initial part of the linear increase, caused by the gravitational contribution and the photon pressure of the optical tweezers of both data sets agree. Only at separations above $350 \mathrm{~nm}$ significant deviations occur, which are caused by the comparatively long readout times of the camera leading to poor statistics in the low-intensity wing of the histogram. For this reason we restrict our analysis to separations smaller than $350 \mathrm{~nm}$ in the following.

The EMCCD camera allows to observe multiple particles at the same time. Further, it enables us to measure scattered intensities from multiple particles at finite flow rates. If the probe particles flow along the wall, their residence time in the field of view, which is the time over which an intensity trace from a given particle can be recorded, is of the order of ten seconds. To infer the sphere-wall interaction potential, the intensity traces from a multitude of particles are recorded and merged into an effective single particle trace. This implies that the trace over time of the scattering intensity of one particle and the sum of the intensity traces of many individual particles over a defined period of time are interchangeable, which is a valid assumption only if all particles are of identical or at least very similar size, shape and optical properties, and if their Brownian motion normal to the interface is uncorrelated.

For the purpose of calculating the effective single-particle intensity trace and discriminating between single particles in the pictures, all particles in the field of view are tracked with a particle tracking algorithm developed by Crocker and Grier [26] (see fig. 2). The code yields the particle's image position on the EMCCD-chip and the intensity at any given time. To compute an effective intensity trace, the recorded particle traces are put through an exclusion filter to remove false hits by checking the mean value and the variance of each individual intensity trace. A selection window for the filter, based on the mean intensity of an individual trace observed during an experiment, is defined, allowing a variation of five percent. The filtering helps to exclude particles close to each other and aggregates. Once the filter process is performed, all selected intensity traces are summed up, $I_{\text {tot }}=\sum_{i} I_{i}$, to yield an effective single particle intensity trace. Once the effective intensity trace is established the standard method, described elsewhere $[20,22]$ can be applied to calculate the potential. However, there are two requirements which have to be met in order to obtain meaningful results: i) the size polydispersity of the probe spheres and the variation of their dielectric properties have to be as small as possible and ii) the illumination intensity has to be homogeneous troughout the field of view.

Further, it is not immediately obvious that Botzmann's law may be used for particles in a flow field to infer the sphere-wall interaction potential from the separation distance probability distribution. Strictly speaking, particles in a shear gradient or in a flow field are not in thermal equilibrium. However, a free particle close to a wall does not experience any force normal to a wall if a flow field parallel to the wall is applied. The argument for this reasoning is as follows: if the particle experienced a normal force, it would follow a trajectory $\Delta \boldsymbol{r}=\boldsymbol{r}\left(t_{0}+t\right)-\boldsymbol{r}\left(t_{0}\right)$, which is influenced by the component normal to the wall. When inverting the direction of time (going from $t$ to $t_{0}$ ) the particle should return to its origin $\boldsymbol{r}\left(t_{0}\right)$. The acting normal force would point in the same direction for both time directions, so that the particle would not return to its original position. This is a violation of time inversibility, showing that there cannot be any normal force for a free particle in a flow field. Without a normal force the probability distribution in the $z$-direction is not affected by the flow field, leaving the particle in thermal equilibrium when looking at the motion perpendicular to the wall only. By this argument, the standard method to calculate a potential is still valid and can be applied to intensity traces acquired with a shear gradient acting on the probe particles.

\subsection{Realization of flow}

Since TIRM measurements are based on scattered intensity fluctuations caused by Brownian motion, any external source of vibrations has to be excluded. This cannot be granted if a pump or a mechanical shear cell is used to generate flow. Therefore we chose to use Earth's gravitational field to generate a pressure difference between the in- and the outlet of a flow cell. For moderate flow rates, a laminar flow inside a flow cell with a defined geometry can be created. In the setup, a glass flow cell is connected to a reservoir by tubes. This reservoir is a syringe or burette filled with the sample solution. The outlet is equipped with a valve to stop the flow completely. If the valve is opened the suspension will flow through the cell creating the desired flow field. At the end of the tubes the flow is regulated by an electronic flow mass controller (Bronkhorst mini Cori Flow) (see fig. 3).

The device is a combination of a Coriolis flow meter and an electronic valve, which measures the mass flow 


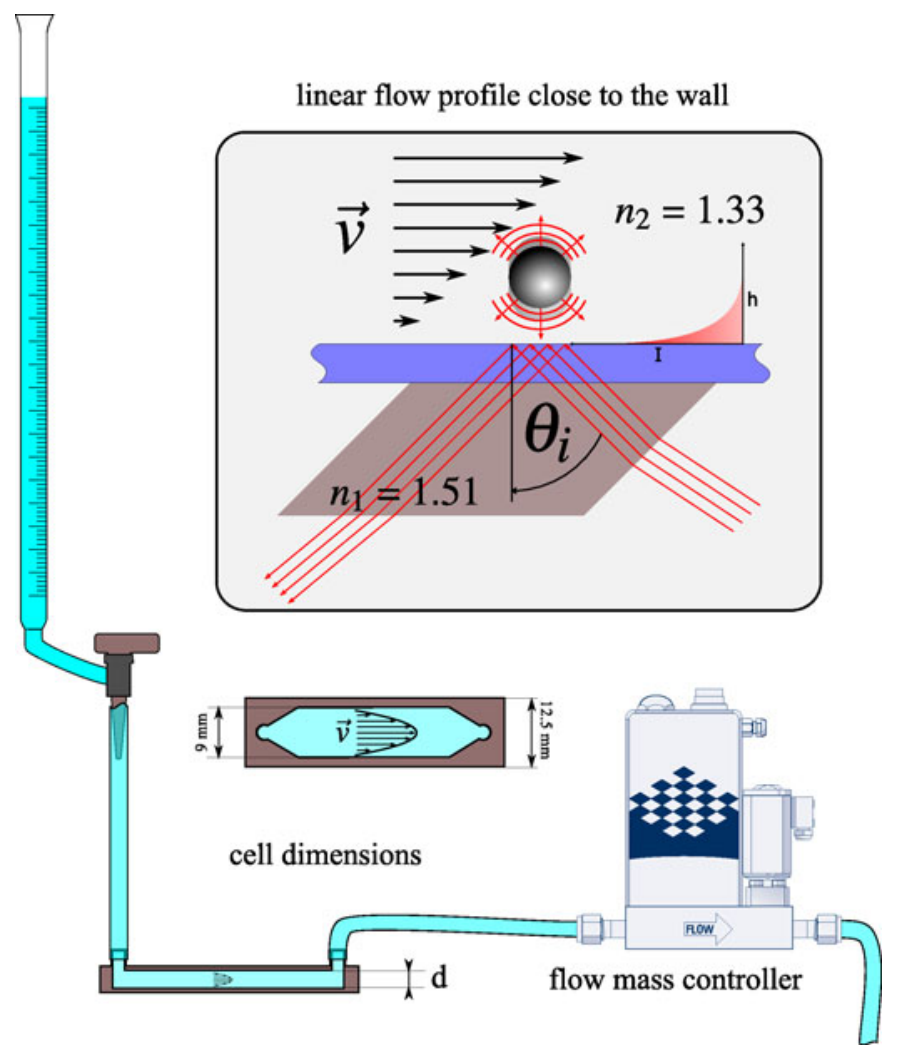

Fig. 3. Sketch of the evanescent illumination and the flow cell with flow mass controller. A laminar flow is produced by using the gravitational field of Earth. The reservoir with the sample solution is placed at a point sufficiently high above the sample stage (about $1 \mathrm{~m}$ ) to create the necessary pressure. The reservoir is connected to a flow cell with a thickness $\mathrm{d}=$ $0.1 \mathrm{~mm}$. The mass flow is controlled and measured by a flow mass controller. The shear rate scales approximately with the mass flow and is approximately constant in the vicinity of the wall.

and controls it to fluctuations smaller than $0.1 \%$. As long as the flow inside the cell is laminar, the flow profile has a parabolic shape and resembles a Poisseuille flow. The velocity profile close to the wall is approximately linear and the shear rate is constant. With this assumption the shear rate may be calculated from the mass flow, $\Phi_{M}$, which is set by the flow meter, and the cell dimensions. The velocity profile of a one dimensional Poisseuille flow is given by

$$
v(z)=\frac{1}{2 \eta} \overbrace{\frac{\mathrm{d} p}{\mathrm{~d} x}}^{\Delta p / L}\left(\frac{d^{2}}{4}-z^{2}\right),
$$

where $v(z)$ is the flow velocity, $z$ the distance from the cell center to the wall, $\Delta p$ the pressure difference between cell's in- and outlet, $L$ the distance between in- and outlet, $d$ the cell thickness and $\eta$ the zero-shear viscosity of the liquid. With

$$
v(z=0)=v_{0}=\frac{1}{8 \eta L} \Delta p d^{2},
$$

eq. (1) can be simplified to

$$
v(z)=v_{0}\left(1-\frac{4 z^{2}}{d^{2}}\right) .
$$

The mass flow, $\Phi_{M}$, through the cell is known from the experiments, which can readily be connected to the velocity in the cell by

$$
\begin{aligned}
\Phi_{M}= & \rho \int_{A} v(z) \mathrm{d} A= \\
& 2 \rho v_{0} \int_{0}^{b} \int_{0}^{d / 2}\left(1-\frac{4 z^{2}}{d^{2}}\right) \mathrm{d} x \mathrm{~d} z=\frac{2}{3} b d \rho v_{0}=\frac{2}{3} A \rho v_{0},
\end{aligned}
$$

where $b$ is the width of a cell $(9 \mathrm{~mm})$ and $\rho$ the mass density of the liquid. The dependence of the shear rate $\dot{\gamma}$ on the distance from the wall and the mass flow is equal to

$$
\dot{\gamma}=\frac{\mathrm{d} v(z)}{\mathrm{d} z}=-v_{0} \frac{8 z}{d^{2}}=-\frac{12 \Phi_{M}}{A \rho}\left(\frac{d / 2-h}{d^{2}}\right),
$$

where $h$ is the distance from the cell wall. This relation is strictly valid only for geometries which are infinitely extended in the direction parallel to the wall and normal to the flow. If the geometry is finite, the flow profile changes with the lateral position inside the field of view. However, a rough calculation shows that the velocity gradient in the lateral direction is smaller by more than two orders of magnitude than in the $z$-direction, and may therefore be neglected, if the field of view is positioned in the center of the flow cell.

\subsection{Sample preparation}

The gibbsite platelets used here have a diameter $\bar{D}=$ $212 \pm 56 \mathrm{~nm}$ and thickness $L=10 \pm 6 \mathrm{~nm}$, as determined by transmission electron microscopy (TEM). They were synthesized according to the procedure described elsewhere $[21,27]$. The platelets are coated with silica [27] to give them a negative surface charge in solution and thereby avoid adhesion to the glass cells. The mass concentration of the stock solution was found to be $c=18 \mathrm{mg} / \mathrm{ml}$ by drying a small amount of suspension and weighing the remaining solid. The mass density of the gibbsite platelets, $\rho_{\text {gibb }}=2.34 \mathrm{~g} / \mathrm{cm}^{3}$, which was checked with a density meter, compares reasonably well with the value from literature for the mass density of gibbsite $\rho_{\text {gibb }}=2.4 \mathrm{~g} / \mathrm{cm}^{3}[28]$. In preparation for the measurement the solution was diluted with purified water $\left(\rho_{R}=18 \mathrm{M} \Omega \mathrm{cm}\right.$, total organic contents less than $2 \mathrm{ppb}$ ) to the desired concentration, while the ionic strength is set by adding $\mathrm{NaOH}$ (Sigma Aldrich $0.1 \mathrm{M}$ ) during dilution, so that the final platelet suspension contains $1 \mathrm{mM} \mathrm{NaOH}$. This high- $p \mathrm{H}$ solvent was chosen to ensure that the glass walls of the sample cell had a sufficiently large charge density to provide electrostatic repulsion. Polystyrene micro spheres $(R=3 \mu \mathrm{m}$, thermo scientific 4206A) are mixed with the sample during the preparation to supply probes for the measurement. 


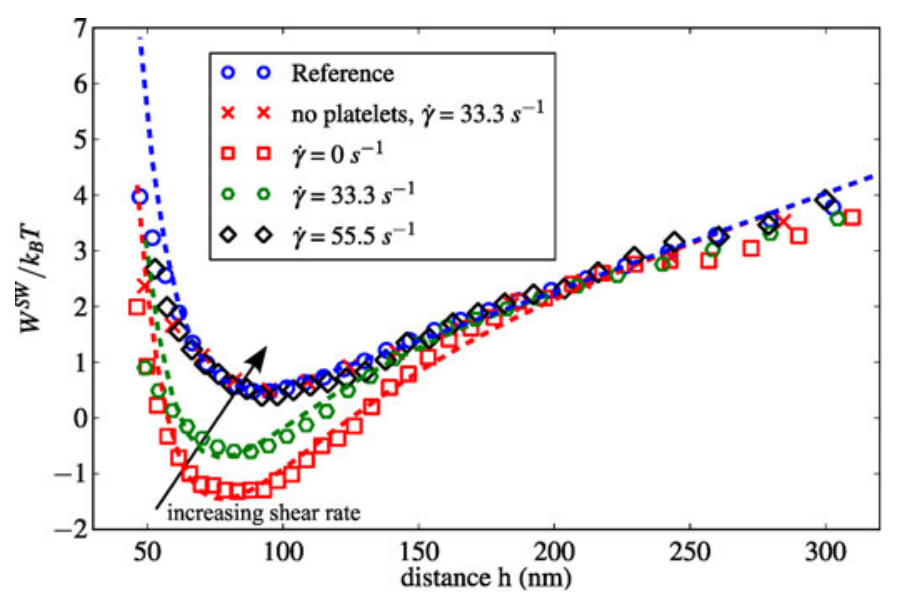

Fig. 4. Interaction potentials as functions of the separation distance between a probe sphere and a glass wall, recorded with and without depletant present $(c=7.2 \mathrm{mg} / \mathrm{ml})$. Data were measured at different shear rates and without shear as indicated. Symbols represent experimental data and dashed lines represent model calculations of the depletion potential caused by polydisperse platelets as discussed in the text.

\section{Results and discussion}

To investigate the influence of a shear flow on the interaction potentials between a probe sphere surrounded by disc shaped depletants and a glass wall we performed TIRM experiments as described in the previous sections. Experiments were conducted employing a shear cell with a thickness of $d=0.1 \mathrm{~mm}$ and setting the penetration depth, $\beta^{-1}$, of the evanescent wave to $150 \mathrm{~nm}$. The illumination time of the camera was set to $10 \mathrm{~ms}$ and 4 times binning of the EMCCD chip was used, to increase the sensitivity and reduce the amount of acquired data.

In fig. 4 we display potential profiles obtained under the following conditions. As a reference the potential between spheres at rest and the wall was measured in the absence of depletant particles. This potential is correctly described by the superposition of an exponentially-decaying branch caused by electrostatic repulsion at small separation distances and a linear branch, which represents the gravitational contribution at large distances. From the slope of the linear part the buoyancy-corrected particle mass is calculated to be $m_{S} \approx 7 \times 10^{-15} \mathrm{~kg}$, which is in good agreement with the value obtained using the particle size and the density difference between polystyrene and water. In the next step, a suspension of the same kind of particles was allowed to flow trough the cell at a mass flow corresponding to a near wall shear rate of $\dot{\gamma}=33.3 \mathrm{~s}^{-1}$. As shown in fig. 4 the potential measured for the flowing spheres coincides with the potential measured at rest, except for the smallest separation distances. This deviation is caused by the variation of the probe spheres' surface charge density, which is due to the fabrication process. The probe spheres are certified to have a very narrow size distribution, which can only be achieved at the cost of a broad charge density variation. Independent experiments at rest without depletant on the same spheres yielded charge densities, which vary over more than one order of magnitude. The third potential, which was measured with the depletant present at a mass concentration of $c=7.2 \mathrm{mg} / \mathrm{ml}$ in a solution at rest, is similar to the first two at low and at high separation distances. However, at intermediate separations a dip of about $3 k_{B} T$ occurs, which is caused by the depletion potential superimposed on the reference profile. Finally, two interaction potentials were measured from a solution containing the probe spheres and the depletants flowing at different velocities corresponding to shear rates of $\dot{\gamma}=33.3 \mathrm{~s}^{-1}$ and $55.5 \mathrm{~s}^{-1}$. The profile obtained at $\dot{\gamma}=33.3 \mathrm{~s}^{-1}$ shows a dip at intermediate separations, which is less deep than that observed for the solution at rest, while this dip is not discernible in the profile measured at $\dot{\gamma}=55.5 \mathrm{~s}^{-1}$.

Together with the experimental data we show model calculations in fig. 4, which are based on the superposition of theoretical predictions in the Derjaguin approximation with an electrostatic repulsion, $W_{\mathrm{er}}^{\mathrm{SW}}$, a gravitational contribution, $W_{\mathrm{g}}^{\mathrm{SW}}$, and a depletion attraction $W_{\mathrm{depl}}^{\mathrm{SW}}[22]$ with

$$
\begin{aligned}
& \frac{W_{\mathrm{er}}^{\mathrm{SW}}}{k_{B} T}=B \times \exp \{-\kappa h\}, \\
& W_{\mathrm{g}}^{\mathrm{SW}}=V_{S} \Delta \rho_{S} g, \\
& \frac{W_{\mathrm{depl}}^{\mathrm{SW}}}{k_{B} T}= \begin{cases}\frac{2}{3} \pi \rho_{N} R \int_{0}^{\infty} D^{2} f(h, D) \Psi(D) \mathrm{d} D, & \text { for } h \leq D, \\
0, & \text { for } h>D .\end{cases}
\end{aligned}
$$

Here $k_{B} T$ is the thermal energy and $B$ is the amplitude of the electrostatic contribution, which is proportional to the charge density. $\kappa^{-1}$ is the Debye screening length, $V_{S}$ the probe sphere volume, $\Delta \rho_{S}$ the density difference between sphere and solvent, $g$ the acceleration of gravity and $\rho_{N}$ the number density of the depletant particles. The integral represents the calculation of an averaged potential taking into account a distribution of the disc diameter $\Psi(D)$ for which we use a logarithmic-normal distribution. The strength of the depletion potential varies with separation distance as

$$
\begin{aligned}
f(h, D)= & -\frac{3}{2} \frac{h}{D}\left(\sin ^{-1} \frac{h}{D}-\frac{\pi}{2}\right) \\
& -\left(1+\frac{1}{2}\left(\frac{h}{D}\right)^{2}\right) \sqrt{1-\left(\frac{h}{D}\right)^{2}} .
\end{aligned}
$$

For the calculation of the curves in fig. 4 the parameters determining the electrostatic contribution were kept constant for all data sets at the values which were determined from a linear least squares fit of eq. (5) to the experimental data set obtained in the presence of depletant at rest, i.e. $B=15.3 k_{B} T, \kappa^{-1}=12.1 \mathrm{~nm}$. The gravitational contribution was calculated from the known sphere radius $R=3 \mu \mathrm{m}$ and the density difference $\Delta \rho_{S}=0.55 \mathrm{~g} / \mathrm{cm}^{3}$. Only the average disc diameter, $\bar{D}$, and the size distribution's standard deviation, $\sigma_{D}$, were varied to produce the best match with the experimental data for those cases 


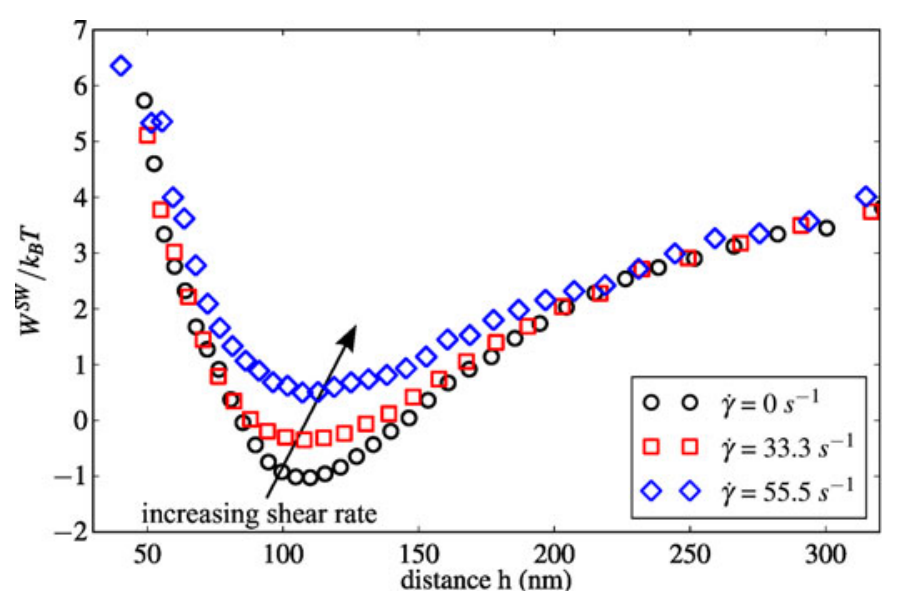

Fig. 5. Interaction potentials as functions of the separation distance between a probe sphere and a glass wall, recorded in the presence of depletant $(c=7.2 \mathrm{mg} / \mathrm{ml})$. Data were measured at the same shear rates as those shown in fig. 4 to verify reproducibility.

where experimental data were obtained from flowing solutions. Without flow, the nonlinear least squares fit yields $\bar{D}=230 \mathrm{~nm}$ and a standard deviation, $\sigma_{D}=40 \mathrm{~nm}$ in very good agreement with the disc size distribution determined independently with TEM. In order to achieve a good agreement with the data measured at $\dot{\gamma}=33.3 \mathrm{~s}^{-1}$ it was necessary to assume $\bar{D}=170 \mathrm{~nm}$ and $\sigma_{D}=30 \mathrm{~nm}$. For the highest applied shear rate, $\dot{\gamma}=55.5 \mathrm{~s}^{-1}$ the experimental data are nearly identical with those of the reference measurement without platelets. Consequently, $\bar{D}$ was chosen to be zero for the model calculation.

The measurements were repeated once more with a new sample with the same depletant concentration, to assure that the observed effect is reproducible. In fig. 5 the experimental results are shown without a reference measurement and without model curves. It is evident that the potential dip gradually disappears with increasing shear rate. For solutions with a lower platelet concentration $(c=6 \mathrm{mg} / \mathrm{ml})$ qualitatively the same effect was observed, but the potential dips are relatively shallow, close to the resolution limit of the instruments. We, therefore, refrain from displaying the data. Surprisingly, for a mass concentration of $c=9 \mathrm{mg} / \mathrm{ml}$ we observe a qualitatively different behavior. The potentials measured in the quiescent state (no shear flow) which are shown in fig. 6 follow the theoretical prediction. As to be expected from higher depletant concentration, the potential well caused by the depletion contribution is more pronounced compared with the data shown in fig. 5 , due to the increased number density of platelets. However, if flow is applied, the depth of the potential well is not affected, which is in clear contrast to the observations with solutions of lower depletant concentration.

According to eq. (5), the depth of the contact value, $\lim _{h \rightarrow 0} W_{\mathrm{depl}}^{\mathrm{SW}}$, of the depletion potential scales with the square of the disc diameter at constant number density, for monodisperse disc shaped depletants. Also for polydisperse discs, the potential depth will vary with the mean

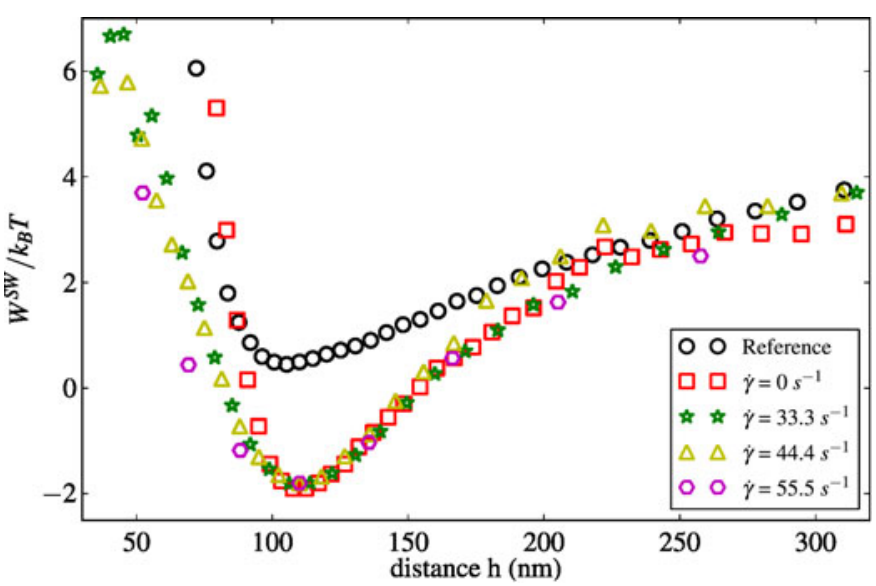

Fig. 6. Interaction potentials as functions of the separation distance between a probe sphere an a glass wall, recorded with and without depletant present $(c=9 \mathrm{mg} / \mathrm{ml})$. Data were measured at different shear rates and without shear as indicated.

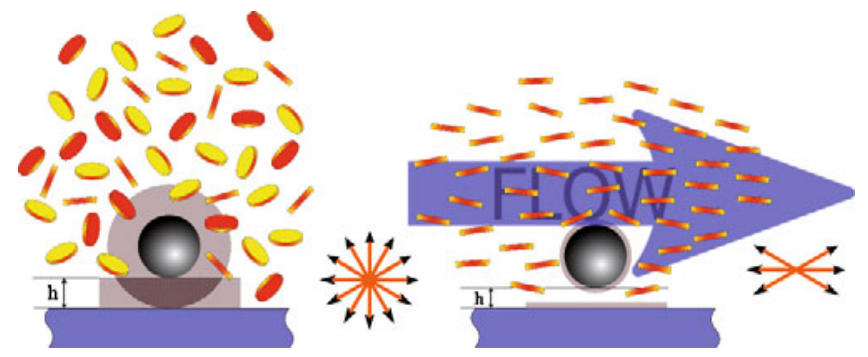

Fig. 7. Cartoon of shear alignment of platelets on depletion interaction. If the volumes around the sphere and above the wall, which are excluded for the centers of mass of the depletants (light gray), overlap (dark gray area), a net force is created, which pushes the sphere towards the wall. If the discs are aligned the excluded volumes shrink and the overlap volume and consequently the depletion force may vanish. The dimensions are not drawn to scale.

disc diameter $\bar{D}$. With the assumption that the potentials measured under flow can still be described by eq. (5), the weakening of the depletion interaction is explained by a reduction of the effective disc diameter with increasing shear rate. This leads us to interpret the observed effects in terms of a shear alignment model as sketched in fig. 7 . As the platelets are subject to a shear gradient, the orientations of the platelets will not be evenly distributed any more, but rather the distribution will peak in the direction of the flow. Under such conditions, the effective diameter of the depleting platelets becomes smaller which consequently shortens the range and weakens the strength of the depletion attraction.

A thorough quantification of the observed trend is not possible due to the lack of a theoretical model. The only available theoretical treatment on a related problem was published by Dzubiella et al. [9], who treated the situation of spherical depletants flowing past two probe spheres at fixed positions in a quiescent viscous solvent. They showed that the depletion forces between the probe spheres strongly depend on the flow velocity of the 
depletant and on the direction of the flow with respect to the vector connecting the centers of the probe spheres. For certain combinations of these parameters they even predicted repulsive barriers in the depletion force profile. Since the situation analyzed in that paper is fundamentally different from the conditions in the present work, we have to limit our data interpretation to the phenomenological picture sketched above. However, there are some issues, which are not covered by this simple picture. The assumption that the flow velocity profile is linear everywhere in the vicinity of the wall is a simplification which might not be justifiable. In the vicinity of the probe spheres the flow field is certainly not laminar but it will be disturbed by the presence of the large particles. However, we do not have the possibility to include this disturbance into the model. Further, it is generally understood that the shear rate required to align a platelet is of the order of or larger than the rotational diffusion coefficient $D_{\text {rot }}$ of a single platelet. For an infinitely thin platelet, Happel and Brenner [29] devised an analytic expression which is used to estimate the minimal shear rate required to align one platelet in solution.

$$
\dot{\gamma} \geq D_{\text {rot }}=\frac{k_{B} T}{(4 / 3) \eta D^{3}}
$$

Calculating the diffusion coefficients for platelets with a diameter of $212 \mathrm{~nm}$ at $T=295 \mathrm{~K}$ in water yields $D_{\text {rot }}=$ $320 \mathrm{~s}^{-1}$, which is larger than the shear rate by an order of magnitude which we had to apply to suppress the depletion interaction. However, it is well known that the dynamics of particles close to interfaces may be drastically slowed down as compared to the free bulk dynamics by the so-called "wall drag" effect. For spherical particles close to planar interfaces, this is theoretically well described [30$32]$ and supported experimentally [33-35]. For non spherical particles there is neither theoretical nor experimental work available. It is however expected from computer simulations that rotational dynamics of rods are slowing down drastically in the vicinity of a flat wall [36]. It is therefore a fair assumption that the rotational dynamics of disc-shaped colloids will be much slower in the vicinity of a wall and the surface of a large sphere. Nevertheless, it remains a task for future theoretical and experimental work to quantify this effect.

Finally, the qualitatively different behavior, which is observed at high disc concentrations, cannot be explained by shear alignment of the depletant alone. At this time we have only two rudimentary arguments to explain this observation. There is experimental evidence that the gibbsite platelets we used as depletant have a tendency to align with their faces towards flat walls [37]. In combination with a shear flow this might lead to a paranematic or an other kind of induced liquid crystalline structure close to the wall. In this scenario, the order parameter of this pseudophase would be highly frustrated by the presence of the probe sphere eventually leading to an elastic force expelling the probe sphere from the ordered structure. In this situation, increasing the share rate would lead to an increase rather than a decrease of the force pushing the probe particle to the wall. The second scenario is based on the observation that suspensions of non-spherical particles in a shear flow show a transition from an alignment region in the shear rate $v s$. concentration plane of their phase diagram to a region where the particles undergo tumbling motions [38]. If in our case, the disc dynamics under shear were dominated by tumbling, the effective dimensions of the discs should not be severely influenced by the flow, and consequently no effect on the depletion interaction would be observed. Both scenarios are merely hypothetical and to prove or discard them will require very sophisticated experiments. These are far beyond the scope of this contribution, where we focus on showing that it is, in principle, possible to tune depletion interactions by flow if non-spherical depletants are used.

\section{Conclusion}

We show experimentally that depletion forces, which are induced by non-spherical depletants, can be tuned by flow fields and eventually switched off at sufficiently high shear rates. We interpret this effect as the consequence of the alignment of the disc-shaped depletants in the shear field which reduces the effective size of the platelets. The influence of a shear flow on the depletion potential vanishes above a threshold of the depletant concentration. It is very likely that similar effects can be achieved by aligning ansitropic, susceptible depletants in high-frequency electric or magnetic fields. Although this work represents a proof of principle only, and we have to leave many open questions to future investigations, we are convinced that this may open a new and very effective route manipulate phase behavior and structure formation of colloidal suspensions, in which depletion interactions play a role.

We thank Jan K.G. Dhont and Pavlik Lettinga for the fruitful discussions. The authors acknowledge financial support from the EU through FP7, project Nanodirect (Grant No. NMP4SL-2008-213948).

Open Access This is an open access article distributed under the terms of the Creative Commons Attribution License (http://creativecommons.org/licenses/by/3.0), which permits unrestricted use, distribution, and reproduction in any medium, provided the original work is properly cited.

\section{References}

1. H.N.W. Lekkerkerker, R. Tuinier, Colloids and the Depletion Interaction (Springer, 2011).

2. S. Asakura, F. Oosawa, J. Polym. Sci 33, 183 (1958).

3. J.C. Conrad, H.M. Wyss, V. Trappe, J. Rheol. 54, 421 (2010).

4. K.R. Purdy, S. Fraden, Phys. Rev. E 70, 061703 (2004).

5. V.J. Anderson, H.N.W. Lekkerkerker, Nature 416, 811 (2002).

6. W.C.K. Poon, J. Phys.: Condens. Matter 14, R 859 (2002). 
7. G.E. Fernandes, D.J. Beltran-Villegas, M.A. Bevan, Langmuir 24, 10776 (2008).

8. R. Fåhraeus, Phys. Rev. 9, 241 (1929).

9. J. Dzubiella, H. Löwen, C.N. Likos, Phys. Rev. Lett. 91, 248301 (2003)

10. K.-H. Lin, J.C. Crocker, A.C. Zeri, A.G. Yodh, Phys. Rev. Lett. 87, 088301 (2001).

11. D. Rudhardt, C. Bechinger, P. Leiderer, Phys. Rev. Lett. 81, 1330 (1998).

12. M. Piech, J.Y. Walz, J. Colloid Interface Sci. 232, 86 (2000).

13. A. Sharma, J.Y. Walz, J. Chem. Soc., Faraday Trans. 92, 4997 (1996)

14. P. Holmqvist, D. Kleshchanok, Peter R. Lang, Eur. Phys. J. E 26, 177 (2008).

15. P. Holmqvist, D. Kleshchanok, P.R. Lang, Langmuir 23 , 12010 (2007)

16. L. Helden, G.H. Koenderink, P. Leiderer, C. Bechinger, Langmuir 20, 5662 (2004).

17. L. Helden, R. Roth, G.H. Koenderink, P. Leiderer, C. Bechinger, Phys. Rev. Lett. 90, 048301 (2003).

18. P.C. Odiachi, D.C. Prieve, Colloids Surf. A 146, 315 (1999).

19. P.R. Lang D. Kleshchanok, R. Tuinier, Langmuir 22, 9121 (2006).

20. D.C. Prieve, Adv. Coll. Interface Sci. 82, 93 (1999).

21. A.M. Wierenga, T.A.J. Lenstra, A.P. Philipse, Colloids Surf. A 134, 359 (1998)

22. C. July, D. Kleshchanok, P.R. Lang, Soft Matter 7, 6444 (2011).
23. Z. Dogic, S. Fraden, Curr. Opinion Colloid Interface Sci. 11, 47 (2006).

24. D. Kleshchanok, M. Heinen, G. Nagele, P. Holmqvist, Soft Matter 8, 1584 (2012).

25. F.M. van der Kooij, K. Kassapidou, H.N.W. Lekkerkerker, Nature 406, 868 (2000).

26. J.C. Crocker, D.G. Grier, J. Colloid Interface Sci. 179, 298 (1996).

27. J.E.G.J. Wijnhoven, Chem. Mater. 16, 3821 (2004).

28. D. Lide, CRC Handbook of Chemistry and Physics, Vol. 91 (Taylor and Francis, 2010).

29. J. Happel, H. Brenner, Low Reynolds number hydrodynamics (Kluwer Academic Publishers, 1963).

30. H. Brenner, Chem. Eng. Sci. 16, 242 (1961).

31. A.J. Goldman, R.G. Cox, H. Brenner, Chem. Eng. Sci. 22, 637 (1967)

32. B. Cichocki, B. Jones, Physica A 258, 273 (1998)

33. Z. Adamcyzk, M. Adamcyzk, T.G.M. Van De Ven, J. Colloid Interface Sci. 96, 204 (1983).

34. B.H. Lin, J. Yu, S.A. Rice, Phys. Rev. E 62, 3909 (2000).

35. P. Holmqvist, J.K.G. Dhont, P.R. Lang, J. Chem. Phys. 126, 044707 (2007).

36. J.T. Padding, W.J. Briels, J. Chem. Phys. 132, 054511 (2010).

37. D. Kleshchanok, A.V. Petukhov, P. Holmqvist, D.V. Byelov, H.N.W. Lekkerkerker, Langmuir 26, 13614 (2010).

38. R.G. Winkler, G. Gompper, J.K.G. Dhont, M. Ripoll, P. Holmqvist, M.P. Lettinga, Phys. Rev. Lett. 101, 168302 (2008). 